\title{
Art Therapy and the Malnourished Brain: The Development of the Nourishment Framework
}

\author{
Article submission for \\ Art Therapy: Journal of the American Art Therapy Association
}

ARTTHERAPY-D-19-00021

Eileen Misluk-Gervase

INDIANAPOLIS, INDIANA

USA

Editor's Note: Eileen Misluk-Gervase, ATR-BC, LPC is an Assistant Professor, Director and Internship Coordinator for the Art Therapy Program at Indiana University Purdue University Indianapolis with Herron School of Art and Design, Indianapolis, Indiana. Correspondence can be directed to the author at emisluk@iupui.edu

This is the author's manuscript of the article published in final edited form as: 
Art Therapy and the Malnourished Brain: The Development of the Nourishment Framework

\author{
Article submission for \\ Art Therapy: Journal of the American Art Therapy Association
}

ARTTHERAPY-D-19-00021

Word Count: 5,276 (6 figures, 1 table) 


\begin{abstract}
Art therapy can be particularly successful in addressing the specific needs of individuals struggling with anorexia nervosa (AN) through the use of the creative process. This article provides an understanding of the effect of malnourishment on the brain for individuals with AN and discusses how their unique needs can be met through the application of the Nourishment Framework. The Nourishment Framework is a structured treatment approach that utilizes the individual components of the Expressive Therapies Continuum (ETC) to address specific clinical needs for those struggling with AN. A case study documents the application of the Nourishment Framework while highlighting the directives and materials used to meet client goals.
\end{abstract}

Keywords: art therapy, anorexia nervosa, malnourished, Expressive Therapies Continuum 


\section{Art Therapy and the Malnourished Brain: The Development of the Nourishment Framework}

The prevalence of eating disorders is truly unknowable because the majority of individuals meeting the clinical criteria never seek treatment or receive a formal diagnosis, (Hart, Granillo, Jorm, \& Paxton, 2011). In 2007, it was estimated that 30 million Americans -20 million females and 10 million males- would meet the clinical criteria for an eating disorder at some point in their lifetime (Hudson, Hiripi, Pope, \& Kessler, 2007). A 2010 study followed a group of 496 adolescent girls for 8 years and found that 13.2\% developed an eating disorder by age 20 (Stice, Martin, Shaw, \& Jaconis, 2009). Although rates of eating disorders have continued to increase across all demographics, a 2014 study showed a more prevalent increase in three groups: males, those from lower socio-economic backgrounds, and older adults (Mitchison, Hay, Slewa-Younan, \& Mond, 2014). Western culture supports disordered eating patterns through harmful dieting and exercise practices and manipulative media messaging about health and wellness, which complicates the treatment and recovery for individuals struggling with eating disorders (Campos, 2004; Culbert, Racine, \& Klump, 2015).

More specifically, between $0.9 \%$ and $2.0 \%$ of females and $0.1 \%$ and $0.3 \%$ of males will develop a form of disordered eating known as anorexia nervosa (AN; Stice \& Bohon, 2012), which is categorized by the restriction of energy intake resulting in low weight, disturbance in body image, and fear of gaining weight (American Psychiatric Association, 2013). Individuals with AN may present with perfectionism, obsessive-compulsiveness, rigidity, treatment resistance, limited affect and emotional expression, anhedonia, alexithymia, and limited social spontaneity (Kaye, 2008). AN can cause cardiovascular and neurological complications, impaired physical development, and a marked increase in suicidality (Chavez \& Insel, 2007). AN 
frequently co-occurs with depression, anxiety, obsessive characteristics, substance abuse disorders, and impairments in social functioning (Chavez \& Insel, 2007; Tagay, Schlottbohm, Reyes-Rodriguez, Repic, \& Senf, 2014). Moreover, individuals struggling with AN tend to register emotional problems as physical signals reporting stomach pain, bowel irregularities, or other unexplained psychosomatic complaints (van der Kolk, 2014). AN presents distinct treatment concerns for clinicians, notably, psychological and physiological effects associated with refeeding and healing the brain and body from significant and long-term malnourishment.

Unlike bulimia nervosa and binge eating disorder where Cognitive Behavioral Therapy (CBT) is identified as the most effective treatment approach, there has been no recommended treatment approach for AN (Dalle Grave, El Ghoch, Sartirana, \& Calugi, 2016; National Collaborating Center for Mental Health, 2004). CBT has been effective in addressing treatment concerns such as anxiety, depression, self-image, and obsessive-compulsive behaviors-all significant factors in AN-but there is little substantial evidence that CBT is effective at addressing the full complexity of AN (Galsworthy-Francis \& Allen, 2014). Building on Hinz's (2006) recommendations that art therapy aids effective treatment and recovery for individuals struggling with AN, this article introduces the Nourishment Framework that helps individuals with AN to be receptive to the dietary requirements and subsequent changes happening to their mind and body.

\section{Malnourished Brain Implications}

Researchers conducting studies of the effects of malnourishment on the AN brain are met with unique hurdles-small sample sizes and the inability to determine baseline brain functioning prior to malnourishment present difficult challenges in attaining significant and generalizable results (Arnold, 2013; Frank, 2011; Kaye, 2008; Rose, Kenney, Rosselli-Navarra, \& Weissman, 
2014). Despite these challenges, studies have been successful in identifying nine distinct areas of the brain that suffer negative effects from malnourishment that correlate with the clinical presentation of individuals with AN: amygdala, hippocampus, somatosensory cortex, cingulate gyrus, prefrontal cortex, insula, parietal lobe, basal ganglia, and the nucleus accumbens (Arnold, 2013). The following offers a brief overview of these nine areas (Figure 1) rather than a comprehensive description of the complex and interconnected nature of the brain and its functions:

- The amygdala is the fear or emotional center of the brain. It identifies threat or danger and alerts the larger system that survival in the form of fight, flight, or freeze may be necessary. The amygdala, which is moderated by the prefrontal cortex, cingulate gyrus, and hippocampus (Arnold, 2013) is involved in food selection and moderating food intake (RajMohan \& Mohandas, 2007). When malnourished, the amygdala serves as a constant sounding alarm, resulting in a brain that is searching for and processing danger.

- When the hippocampus, the memory center where long-term memories are stored and linearly organized (Hass-Cohn, et al., 2014; Lusebrink, 2004; RajMohan \& Mohandas, 2007) is malnourished, an individual may experience disorganization, therefore making it difficult to receive and organize new information (Arnold, 2013).

- When the somatosensory cortex, known to be responsible for body awareness and organization of sensory stimuli experiences malnourishment, it becomes unable to mediate the experience of the body, resulting in distortions of the self (Arnold, 2013).

- Malnourishment in the cingulate gyrus, which detects errors in attention and emotional processing, attunes to others, and fosters emotional connections (Arnold, 2013; 
RajMohan \& Mohandas, 2007) inhibits the capacity for interpersonal connection, including sensitivity and communication with others (Arnold, 2013).

- The prefrontal cortex processes self-construct, self-reflective consciousness, complex social functions, abstract thinking, cognitive flexibility, source and working memory, sustained and directed attention, and planned and spontaneous creative activity (Dietrich, 2004). It is suggested that the "prefrontal cortex is active in the capacity to appropriately or inappropriately restrict food, possibly via heightened fear-related activation and anxious cognitions that drive food restrictions" (Frank, 2011, p. 529). Due to malnourishment, the prefrontal cortex is unable to filter external and internal experiences, while excessive amygdala activity leads to impaired decision-making.

- Malnourishment causes immobilization of the insula, which is responsible for transmitting information throughout the brain and processing interoceptive awareness, that is, the sense of the body in space and the integrative process between cognitive and affective experiences (Arnold, 2013). As a result, the brain is unable to prioritize information and a limited concept of self and environment develops.

- The parietal lobe is responsible for world awareness, mathematical tasks and managing the details needed for global understanding. It also connects to the somatosensory cortex, integrates spatial information from the visual cortex (Lusebrink, 2004), and processes bodily experiences (Christian, 2008). Malnourishment of the parietal lobe results in excessive attention to detail and facts to a degree that it warps the experience of the self in the world (Arnold, 2013).

- The basal ganglia are responsible for executive functions and behaviors, such as implicit learning, automatic behaviors and movements, and processing motor information from 
the somatosensory cortex (Lusebrink, 2004). Malnourishment to the basal ganglia produce patterns of perfectionism and compulsory behaviors (Arnold, 2013).

- Malnourishment in the nucleus accumbens, which moderates the reward and punishment pathway can create imbalance and lead to irrational pleasure-seeking behaviors without awareness of the consequences (Arnold, 2013).

Understanding the functions of the brain in malnourishment provides a more comprehensive understanding of the diagnostic profile of AN. From these findings, an understanding of the potential effects of art making and creativity on the brain were explored. The following overview is largely composed of theorized neurologically based research and is aimed at finding connections between the areas and functions of the compromised brain and art making in a therapeutic setting.

\section{Neuroscience and Art Therapy: Expressive Therapies Continuum Implications}

Art therapy is an active therapeutic process that integrates the mind and body, allowing an individual to uncover, explore, and process emotional content through art making. Art therapy often pairs fear-arousing emotions with positive new sensory experiences as a means of coping, regulating, and integrating (Hass-Cohen, 2008). Art therapists encourage spontaneous engagement, support attention and logical understanding, and create a holding space for overwhelming experiential states (Shore, 2014). Such assumptions may be validated by understanding how creativity provides insight into the ways in which art making may activate neural networks. Creative thinking requires methodological problem solving, organization, cognitive flexibility, abstract thinking, planning, willed action, and source and working memory (Dietrich, 2004; Ellamil, Dobson, Beeman, \& Christoff, 2012). Engaging in creative activities 
heightens a person's "ability to engage in contradictory modes of thought, including cognitive, affective, deliberate, and spontaneous processing” (Ellamil, et al., 2012, pp. 1791-1792).

Creativity activates three brain networks-Executive Attention, Imagination, and Salience-which house four of the nine areas that suffer negative effects from malnourishment (Gotlieb, Hyde, Immordino-Yang, \& Kaufman, 2018). The Executive Attention Network includes the outer regions of the prefrontal cortex and parietal lobe, beneath which lies the hippocampus, which moderates the amygdala (Gotlieb, et al., 2018). The Imagination Network includes the inner regions of the prefrontal cortex, temporal lobe, parietal cortex, and Broca's area. This network constructs mental simulations from past experiences by employing the same processes used in remembering, future planning, and imagining alternative perspectives for the present (Gotlieb et al., 2018). The Salience Network which consists of the dorsal anterior cingulate cortices and anterior insular, is activated in reward and punishment pathways and body distortions (Arnold, 2014; Gotlieb et al., 2018).

Further exploration of brain networks, identified that the basal ganglia and the nucleus accumbens may be activated as part of the dorsal and ventral visual stream as a result of engagement in visual, sensory, and creative processes (Hass-Cohen \& Loya, 2008; Lusebrink, 2004; Lusebrink \& Hinz, 2016). The dorsal visual stream, or the where-how pathway, integrates vision and action and detects movement and location (Hass-Cohen \& Loya, 2008). The ventral visual stream, or the what pathway, responds to shapes, meaning, form, color, and brightness (Hass-Cohen \& Loya, 2008). Hass-Cohen, Finlay, Carr, and Vanderlan (2014) identifed, "The insula and medial prefrontal cortex mediate emotional awareness, as characterized by creativity and the ability to sense the here and now" (p. 72). Each of these structures are involved during 
the act of subjective decision-making, such as choosing colors, shapes, and forms in meaningful image making.

Due to the minimal research on the neuroscience of art therapy, the field relies on theories of visual perception and imagery to understand the effects of art therapy on the brain (Lusebrink, 2014). The Expressive Therapies Continuum (ETC) is a conceptual model that postulates the effects of art making and the creative processes involved in art therapy (Hinz, 2009; Lusebrink \& Hinz, 2016). The ETC organizes media and expression on three levels of complexity: Kinesthetic/Sensory, Perceptual/Affective, Cognitive/Symbolic, and a fourth level, Creative, which is conceptualized to cross all other levels (Lusebrink, 2004; Lusebrink \& Hinz, 2016). This organization reflects "increasing complexity of visual expression and information processing, including the increasing complexity of imagery formation" (Lusebrink \& Hinz, 2016, p. 9).

It is suggested that the Kinesthetic/Sensory component processes preverbal information and somatosensory experiences in the right hemisphere of the limbic system (Hinz, 2009). The Sensory component mediates the internal and external sensations that occur with media interaction (Hinz, 2009) while the Kinesthetic component decreases awareness of sensory experiences (Lusebrink \& Hinz, 2016). The Perceptual component may initiate changing one's point of view due to an emphasis on formal elements of art making (e.g., line, color, form, direction), nonverbal communication, and cognitive restructuring (Hinz, 2009). The Affective component supports identification, amplification, discrimination, and expression of emotions (Hinz, 2009). The Cognitive component incorporates planning, decision making, and intentional and deliberate processes that integrate both past and present imagery (Hinz, 2009). The Symbolic component focuses on intuitive and personal symbol formation, metaphor, and the expression of 
self through symbols (Hinz, 2009). The Creative level can be present within each component and functions as an integrating factor within the ETC (Hinz, 2009).. Based on the conceptualization of the foregoing components, it may be suggested that the following areas of the brain may be activated: amygdala, hippocampus, thalamus, somatosensory cortex, posterior cingulate cortex that includes the cingulate gyrus, prefrontal cortex, insula, and parietal lobe (Hinz, 2009; Lusebrink, 2004; 2014; Lusebrink \& Hinz, 2016).

An integration of the research above suggests that all nine areas of the malnourished brain may be engaged in art therapy for therapeutic benefit. Table 1 demonstrates an alignment of this combined research on the nine areas of the malnourished brain with the individual ETC components. Based on these findings, it is proposed that art therapists are uniquely equipped to meet the needs of AN by supporting the malnourished brain through the use of art materials and creative processes, along with verbal and non-verbal therapeutic approaches. Art therapy engages clients in an active therapeutic process that helps to build tolerance to the psychological and physiological processes associated with refeeding and eating disorder recovery.

\section{Nourishment Framework}

The Nourishment Framework is a structured treatment approach that utilizes the individual components of the ETC to support the unique needs of individuals with AN (Figure 2). It is based on my clinical experience and the patterns I noticed in therapuetic needs and challenges that occurred in the early, mid, and termination phases of treatment. As part of their treatment, client are provided with a meal plan that routinely requires meals and snacks throughout the day with increasing amounts as treatment progresses. For many clients, these increasing dietary requirements cause psychological and physical stress. I began to utilize the ETC has a means to organize directives and media to address the concerns at these stages. At the 
same time, I integrated current research on the effects of malnourishment on the brain and how this effects treatment readiness, compliance, and outcomes. As a result, I developed a framework that takes into consideration the effects of the malnourished brain in treatment, clinical experience of how the brain and subsequent thoughts and behaviors change as a result of nutrition, and how art making may impact the thoughts and behaviors of individuals with AN. By understanding the presenting issues as a result of malnourishment, the Nourishment Framework has the potential to aid art therapists in selecting directives and materials that support and strengthen brain functions as it heals, while avoiding those that have the potential to trigger or exacerbate concerns. Body-based interventions were scaffolded throughout art therapy with the focus on reducing anxiety early in treatment, building tolerance and acceptance mid treatment, and exploring and developing an appreciation for the body towards the end. Although each stage is conceptualized separately, as clients move through the continuum and associated processes, they are engaging in the integrative Creative component (Hinz, 2009). The Creative component has the potential to engage all areas of the malnourished brain within a single session and throughout treatment.

The following case study demonstrates an application of the Nourishment Framework. Catherine (pseudonym), a college-aged female and former athlete, was diagnosed with AN and generalized anxiety disorder. Nine months prior to seeking treatment, Catherine began engaging in restrictive eating patterns, which led to significant weight-loss. When she entered outpatient treatment, Catherine had a body mass index (BMI) of 16. As a result, it was recommended that she enter an inpatient facility for more intensive treatment but because she had strong familial support and personal dedication to her treatment, it was deemed clinically appropriate for her to 
receive outpatient services. These included weekly sessions with a psychologist, dietitian, and art therapist, as well as monitoring by a physician.

Catherine participated in art therapy for two years. As part of the psychosocial intake, Catherine was asked to introduce herself through collage. The minimally complex structured directive and resistive media provided external organization and containment. At this stage of AN treatment, the malnourished brain often needs clear direction with limited decision-making requirements and familiar and easy to use materials. Catherine was provided magazines, pre-cut collage images, liquid glue, glue stick, scissors, and a 12 x 18 piece of white paper. Catherine adhered torn images from magazines to the paper using a glue stick. Her collage encompassed approximately $25 \%$ of the paper with all images clustered in the bottom right corner. Catherine's collage indicated television shows, animals, and activities that she enjoyed. While constructing her collage, she answered questions but otherwise was quiet and did not converse. Based on this intake, Catherine's therapeutic goals included: identifying eating disorder thoughts, beliefs, and behaviors; increasing self-esteem and body awareness; and managing psychological and physiological responses to stress and refeeding.

\section{Perceptual component}

The Nourishment Framework begins with the Perceptual component because clinical experience indicated that a client's transition into treatment was best supported by beginning with organizing principles . A client explores forms and shapes with visual harmony and balance, challenging the brain to create order out of chaos (Hinz, 2009). It aims to reduce symptoms of anxiety, exhaustion, impaired decision-making, and limited cognitive functioning through stimulation of the prefrontal cortex, via the ventral and dorsal visual streams (Lusebrink, 2016; Lusebrink \& Hinz, 2016), insula, and hippocampus. 
In the session following the psychosocial intake, I provided Catherine with a 12 " x 18 " piece of paper with a circle drawn in pencil. At this stage, Catherine was given a minimally complex structured directive and easy to use media. "Place the pencil on the circle. Draw a continuous line on the paper until I say stop. Return the line to the edge of the circle. Using these supplies [markers and/or colored pencils] fill in the shapes with color and pattern." When Catherine started the directive, she chose markers and was seated at a table, but eventually moved to the floor where she spread her body out and created art in several different positions including laying on her stomach, sitting on knees, cross-legged, and legs stretched long. This shift in art making position correlated with a shift in the use of therapeutic and art making space. Her repetitious movements along with pattern making and organization attendant to the directive reduced her symptoms of anxiety including racing thoughts and body discomfort.

Catherine worked on this mandala for several sessions and invited me to work alongside her. The perceptual focus on formal elements of line, color, and pattern provided containment of affect that allowed for creativity and collaboration (as demonstrated in Figure 3). The design provided a safe structure that encouraged creativity without complex decisions regarding media, design, or artistic processes that perhaps could overwhelm the malnourished brain. And, through collaboration and creativity, an environment was built that was conducive to early treatment discussions regarding weight gain, eating disorder thoughts and beliefs, and social isolation.

\section{Kinesthetic component}

The Kinesthetic component helps manage the bodily experience of refeeding and weight restoration by decreasing arousal and tension. Catherine indicated readiness for this stage due to stated discomfort and anxiety during refeeding and her utilizing the therapeutic space for art making. The Kinesthetic component began with an unstructured low complexity directive, 
which increased a certain amount of anxiety but also made the task achievable with fine motor movements, "Play with the clay and see what objects you create." The media, modeling clay, was selected to discharge energy, support repetitive movements, provide sensory feedback, and offer an opportunity for self-soothing. The session included creating, destroying and recreating objects, as well as mixing the modeling clay to make marbleized colors and new shades. This process repeated over several sessions and the familiarity of the material allowed Catherine to create recognizable objects that she kept and baked, demonstrating a commitment to her creations.

In the final sessions of the Kinesthetic component, Catherine was directed to explore wet on wet painting with the goal of engaging gross motor movement. I provided her a 14 " x 16 " piece of watercolor paper and water-based inks. The directive was to "fill the paper with color and explore how the inks move." Catherine was encouraged to use large body movements to move the paper and therefore the inks. Over the course of several sessions, the directive challenged her to build tolerance with and work through uncertainty, unpredictability, and anxiety. This process increased the risk of perceived failure, although the low complexity made the task achievable and safe to begin exploration of moving the body in space.

\section{Cognitive component}

The Cognitive component may support increased attention and energy, problem solving, abstract thought processes, concrete representations, and engagement in multi-step processes (Hinz, 2009). It also provides a space to gain reflective distance, intellectualize complex emotions, explore and challenge cognitive and somatic distortions, and identifies internal and external strengths. Catherine's participation in both fine and gross motor movements combined 
with her ability to tolerate emotions evoked during the creative process indicated that she was ready to explore emotions connected to her eating disorder and self-esteem.

I gave Catherine a high complexity, unstructured directive balanced with resistive materials: develop a story using the painting created during the Kinesthetic component, decorative papers, and markers. Catherine's story and artwork focused on the journey of a character named Soki, who was looking for a place where she belonged (Figure 4). This story, which developed over several sessions, paralleled her increasing articulation of her feelings around identity and demonstrated the ability to communicate through metaphor.

\section{Affective component}

The Affective component supports the expression and discrimination of feelings, as well as the development of urge tolerance skills. Urge tolerance is the ability to mediate emotional rises, peaks, crests, and recessions. For clients with AN, placing the Cognitive component before the Affective component helps build an understanding of emotions prior to engaging in the sensations of those emotions. Based on the needs of a client, a therapist may fluctuate between the Cognitive and Affective components to help the client build urge tolerance skills. Catherine's psychological growth indicated that she was ready to move to the Affective component.

Catherine was quiet and reserved; she did not display intense emotions during her sessions but reported having them outside of therapy. She consistently reported that she experienced overwhelming anxiety that presented as racing thoughts, psychosomatic responses, and sleep disturbances. Based on her needs, I provided Catherine a piece of 12" x 18" white paper, tissue paper, and liquid glue with the following direction: "Collage the emotions that are underneath the anxiety by tearing colors of tissue paper for each emotion and layering them onto this paper." The structured high complexity directive allowed Catherine to connect with her 
emotions and represent them through color and occupying space. Over several sessions, Catherine filled the entire paper with a spectrum of maroons, blues, and golds in order to identify that fear, and particularly fear of failure, was the primary emotion underlying her anxiety. This fear of failure was the impetus behind her perfectionism, especially around academics and she managed this fear by restricting nutrition, over-exercising, and limiting social interactions.

\section{Sensory component}

The goals of the Sensory component are to recognize and tolerate internal and external stimulation. At this stage in treatment, Catherine's ability to tolerate her exploration of emotions indicated that she was ready to move to this component as she had developed the skills necessary to tolerate the exploration of body based sensations. Each of Catherine's sessions in this component began with a guided visualization where she was asked to identify an emotion and locate that emotion in her body. Through the use of breathing techniques, Catherine worked to build tolerance to the sensation of that emotion.

Catherine was not interested in engaging in an immersive arts-based sensory experience, so origami was used as an alternate experience that supported processing the visualization and breathing exercises. She explored traditional origami paper, textured papers, and tissue paper through folding, rolling, twisting, and weaving. While exploring origami, she vacillated between the Cognitive component where she was learning, following directions, and creating concrete representations, and the Sensory experience of the different textured papers. At this time, Catherine's physician approved her to engage in meaningful movement, including hiking. I asked to practice thebreathing and visualization exercises while hiking. During these excursions, I asked her to find an object from nature that she connected with and that she could incorporate into a final artwork as a symbol of her journey. These hikes served as an opportunity to connect 
Kinesthetic movement and internal and external stimulations to emotional sensations outside of the therapeutic space. Catherine successfully translated the therapeutic work in session into these hikes and reported that she was able to remain connected to her body and the accompanying sensations. Figure 5-a wall hanging-was composed of selected flowers from various sessions and the object from nature.

\section{Symbolic component}

The Symbolic component requires focus and attention, organization of multi-layered concepts, problem solving, and metaphoric thinking and is most effective in the later stages of recovery as the refeeding process is complete. It illuminates intuition and idiosyncratic thoughts that lead to symbol formation. After 18 months of treatment, Catherine was successfully navigating her recovery-she was thriving in school, developing her social network of friends, meeting meal goals and food challenges, and maintaining her weight.

Catherine was given a high complexity unstructured directive to "create a symbol that represents the recovered self and use printmaking materials to carve and print this symbol.” The process integrated fluid and resistive media and supported an integration of Cognitive and Perceptual aspects of decision-making and design execution, as well as Sensory and Kinesthetic aspects of carving, and the Affective and Symbolic component of rendering a personally meaningful image. Catherine spent several sessions discussing images, symbols, and their relation to her recovery. Once she selected one, she traced and transferred it onto a speedy block

for carving. She spent numerous sessions carving, printing, and re-carving her symbol (Figure 6). Catherine's symbol represented her intelligence, love of learning, and relationships to those around her. The identification and creation of her symbol demonstrated the successful completion of her therapeutic goals and recovery from her eating disorder. 


\section{Conclusion}

The Nourishment Framework offers art therapists a means to understand and treat the psychological, neurological and behavioral needs of individuals struggling with AN.

Additionally, formalizing the framework aids in researching the potential benefits of this approach. With the growing body of knowledge about the brain's role in mental illness, clinicians will be able to use this framework as a guide to develop more comprehensive treatment recommendations in the field of eating disorders.

\section{References}

American Psychiatric Association. (2013). Diagnostic and statistical manual of mental disorders: DSM-5 (5th ed.). Washington, DC: Author.

Arnold, C. (2013). Decoding anorexia: How breakthroughs in science offer hope for eating disorders. New York, NY: Routledge.

Campos, P.F. (2004). The obesity myth: Why America's obsession with weight is hazardous to your health. New York, NY: Gotham Books.

Chavez, M., \& Insel, T.R. (2007). Eating disorders: National Institute of Mental Health's perspective. American Psychologist, 62(3), 159-166. doi: 10.1037/0003-066X.62.3.159

Christian, D. (2008). The cortex: Regulation of sensory and emotional experience. In N. HassCohen \& R. Carr (Eds.), Art therapy and clinical neuroscience (pp. 62-75). Philadelphia, PA: Jessica Kingsley.

Culbert, K. M., Racine, S. E., \& Klump, K. L. (2015). Research review: What we have learned about the causes of eating disorders - a synthesis of sociocultural, psychological, and biological research. Journal of Child Psychology and Psychiatry, 56(11), 1141-1164. doi:10.1111/jcpp. 12441 
Dalle Grave, R., El Ghoch, M., Sartirana, M., \& Calugi, S. (2016). Cognitive behavioral therapy for anorexia nervosa: An update. Current Psychiatry Reports, 18(1), 2. https://doi.org/10.1007/s11920-015-0643-4

Dietrich, A. (2004). The cognitive neuroscience of creativity. Psychonomic Bulletin \& Review, 11(6), 1011-1026. doi:10.3758/BF03196731

Ellamil, M., Dobson, C., Beeman, M., \& Christoff, K. (2012). Evaluative and generative modes of thought during the creative process. NeuroImage, 59(2), 1783-1794. doi:10.1016/j.neuroimage.2011.08.008

Frank, G.K.W. (2011). Brain circuitry models in eating disorders. Psychiatric Annals, 4l(11), 526-531. doi:10.3928/00485713-20111017-05

Galsworthy-Francis, L., \& Allan, S. (2014). Cognitive Behavioural Therapy for anorexia nervosa: A systematic review. Clinical Psychology Review, 34(1), 54-72. doi:10.1016/j.cpr.2013.11.001

Gotlieb, R. J. M., Hyde, E., Immordino-Yang, M. H., \& Kaufman, S. B. (2018). Imagination is the seed of creativity. In J.C. Kaufman \& R. J. Sternberg (Eds.), The Cambridge handbook of creativity (pp. 709-731). New York, NY: Cambridge University Press.

Granger, A. M. (2019). Neuroscience of the Nourishment Framework [digital image]. Herron School of Art and Design, IUPUI, Indianapolis, IN.

Graves-Alcorn, S., \& Kagin, C. (2017). Implementing the Expressive Therapies Continuum: A guide for clinical practice. New York, NY: Routledge.

Hart, L. M., Granillo, M. T., Jorm, A. F., \& Paxton, S. J. (2011). Unmet need for treatment in the eating disorders: A systematic review of eating disorder specific treatment seeking 
among community cases. Clinical Psychology Reviews, 31(5), 727-735.

doi:10.1016/j.cpr.2011.03.004

Hass-Cohen, N. (2008). Partnering of art therapy and clinical neuroscience. In N. Hass-Cohen \& R. Carr (Eds.), Art therapy and clinical neuroscience (pp. 21-42). Philadelphia, PA: Jessica Kingsley.

Hass-Cohen, N., Clyde Finlay, J., Carr, R., \& Vanderlan, J. (2014). “Check, change what you need to change and/or keep what you want": An art therapy neurobiological-based trauma protocol. Art Therapy: Journal of the American Art Therapy Association, 31(2), 69-78. doi:10.1080/07421656.2014.903825

Hass-Cohen, N. \& Loya, N. (2008). Visual system in action. In N. Hass-Cohen \& R. Carr (Eds.), Art therapy and clinical neuroscience (pp. 92-110). Philadelphia, PA: Jessica Kingsley.

Hinz, L. (2009). Expressive therapies continuum: A framework for using in art therapy. New York, NY: Routledge.

Hinz, L.D. (2006). Drawing from within: Using art to treat eating disorders. Philadelphia, PA: Jessica Kingsley.

Hudson, J. I., Hiripi, E., Pope, H. G., \& Kessler, R.C. (2007). The prevalence and correlates of eating disorders in the National Comorbidity Survey Replication. Biological Psychiatry, 61(3), 348-358. doi:10.1016/j.biopsych.2006.03.040

Kaye, W. (2008). Neurobiology of anorexia and bulimia nervosa. Physiology \& Behavior, 94(1), 121-135. doi:10.1016/j.physbeh.2007.11.037

Lusebrink, V. (2014). Art Therapy and the neural basis of imagery: Another possible view. Art Therapy: Journal of the American Art Therapy Association, 31(2), 87-90. doi:10.1080/07421656.2014.903828 
Lusebrink, V. (2004). Art therapy and the brain: An attempt to understand the underlying processes of art expression in therapy. Art Therapy: Journal of the American Art Therapy Association, 21(3), 125-135. doi:10.1080/07421656.2004.10129496

Lusebrink, V., \& Hinz, L. (2016). The Expressive Therapist Continuum as a framework in the treatment of trauma. In J. King (Ed.), Art therapy, trauma and neuroscience: Theoretical and practical perspectives (pp. 42-66). New York, NY: Routledge.

Mitchison, D., Hay, P., Slewa-Younan, S., \& Mond, J. (2014). The changing demographic profile of eating disorder behaviors in the community. BMC Public Health, 14(1), 943. doi:10.1186/1471-2458-14-943

National Collaborating Centre for Mental Health (Ed.). (2004). Eating disorders: Core interventions in the treatment and management of anorexia nervosa, bulimia nervosa and related eating disorders ; National Clinical Practice Guideline No. CG9. Leicester: British Psychological Society [u.a.] Retrieved from https://www.ncbi.nlm.nih.gov

RajMohan, V., \& Mohandas, E. (2007). The limbic system. Indian Journal of Psychiatry, 49(2), 132-139. Retrieved from http://www.indianjpsychiatry.org

Shore, A. (2014). Art therapy, attachment, and the divided brain. Art Therapy: Journal of the American Art Therapy Association, 31(2), 91-94. doi:10.1080/07421656.2014.903827

Stice, E. \& Bohon, C. (2012). Eating disorders. In T. Beauchaine \& S. Linshaw (Eds.), Child and Adolescent Psychopathology (2nd ed., pp. 715-738). Hoboken, NJ: John Wiley \& Sons.

Stice, E., Marti, C. N., Shaw, H., \& Jaconis, M. (2009). An 8-year longitudinal study of the natural history of threshold, sub-threshold, and partial eating disorders from a community sample of adolescents. Journal of Abnormal Psychology, 118(3), 587-597. doi:10.1037/a0016481 
Tagay, S., Schlottbohm, E., Reyes-Rodriguez, M. L., Repic, N., \& Senf, W. (2014). Eating disorders, trauma, ptsd, and psychosocial resources. Eating disorders, 22(1), 33-49. doi:10.1080/10640266.2014.857517

Van der Kolk, B. A. (2014). The body keeps the score: Brain, mind, and body in the healing of trauma. New York, NY: Penguin Books. 


\section{List of Figures and Tables}

Figure 1. Image of the brain with the identified areas affected by malnourishment (Granger, 2019). Each area is labeled with the name and primary function. In italics, the resulting effect of malnourishment is noted.

Figure 2. Visual representation of the Nourishment Framework including the conceptualized benefits of the components of the ETC and the arrows point to the therapeutic focus within each component.

Figure 3. Mandala created in the Perceptual component.

Figure 4. Excerpts of the book created in the Cognitive component.

Figure 5. Origami flowers and stars created in the Sensory component.

Figure 6. Self-symbol image carved and printed in the Symbolic component.

Table 1. Alignment of the Areas of the Malnourished Brain and the ETC 


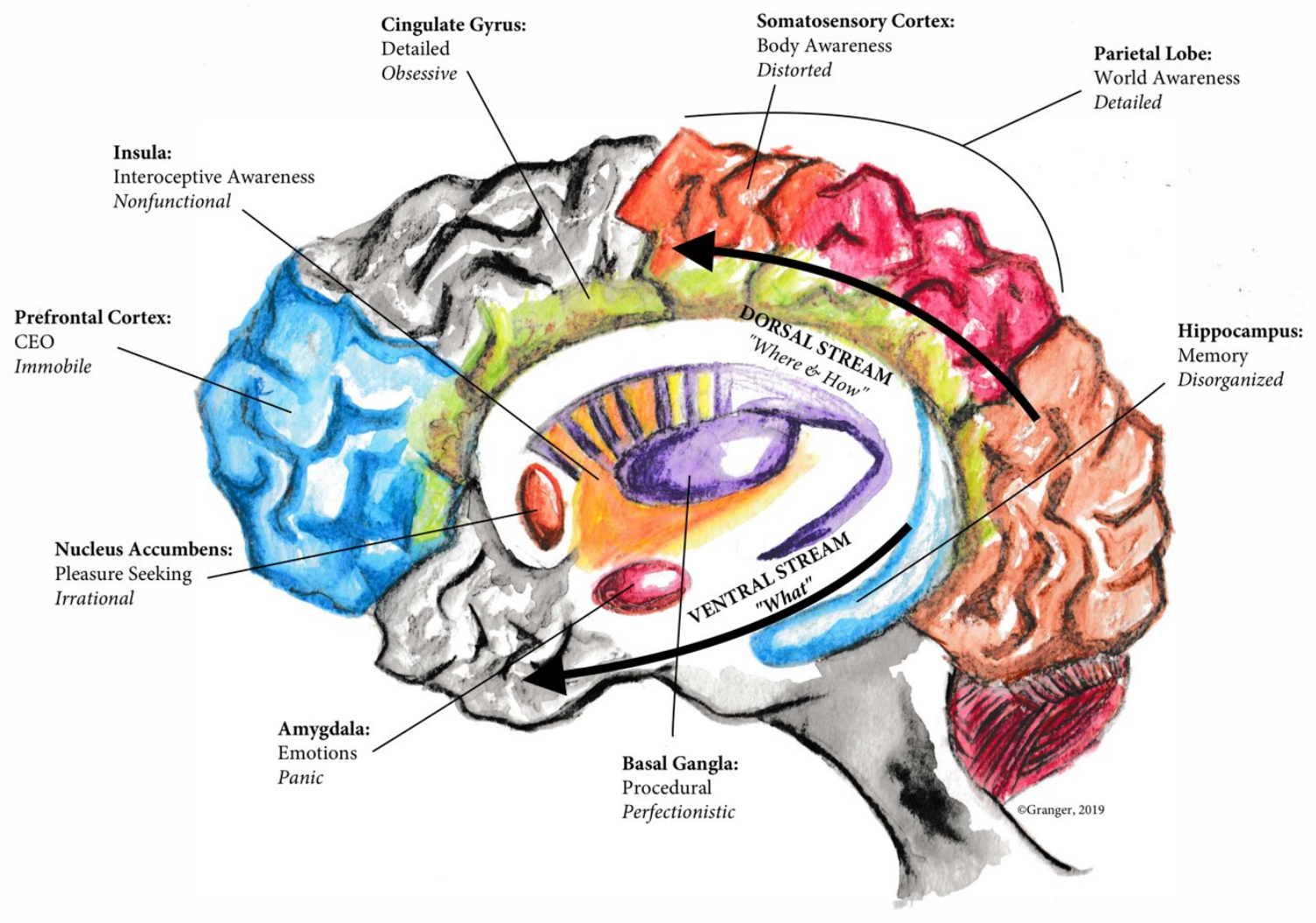

Figure 1. Image of the brain with the identified areas affected by malnourishment (Granger, 2019). Each area is labeled with the name and primary function. In italics, the resulting effect of malnourishment is noted. 


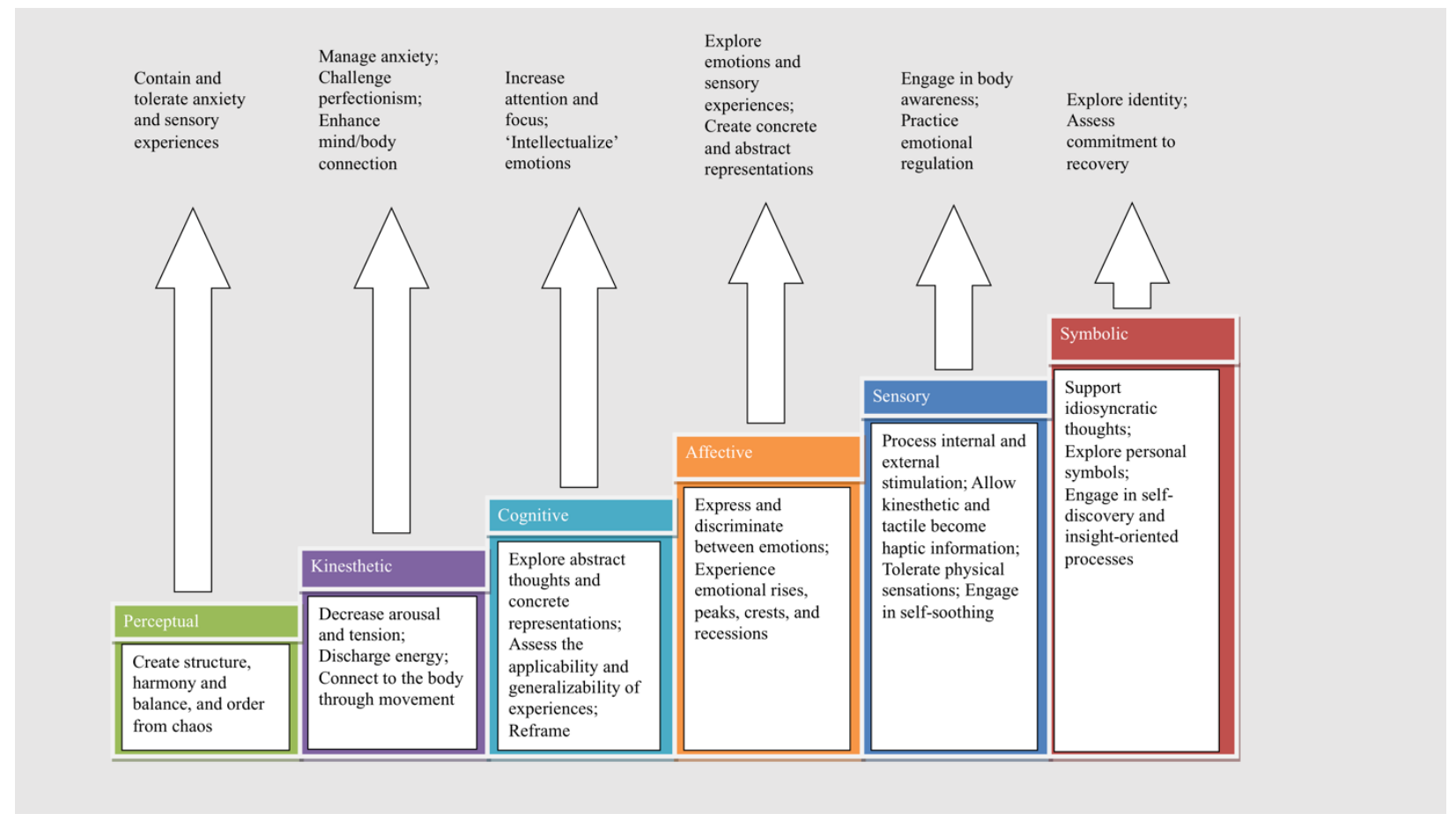

Figure 2. Visual representation of the Nourishment Framework including the conceptualized benefits of the components of the ETC and the arrows point to the therapeutic focus within each component. 


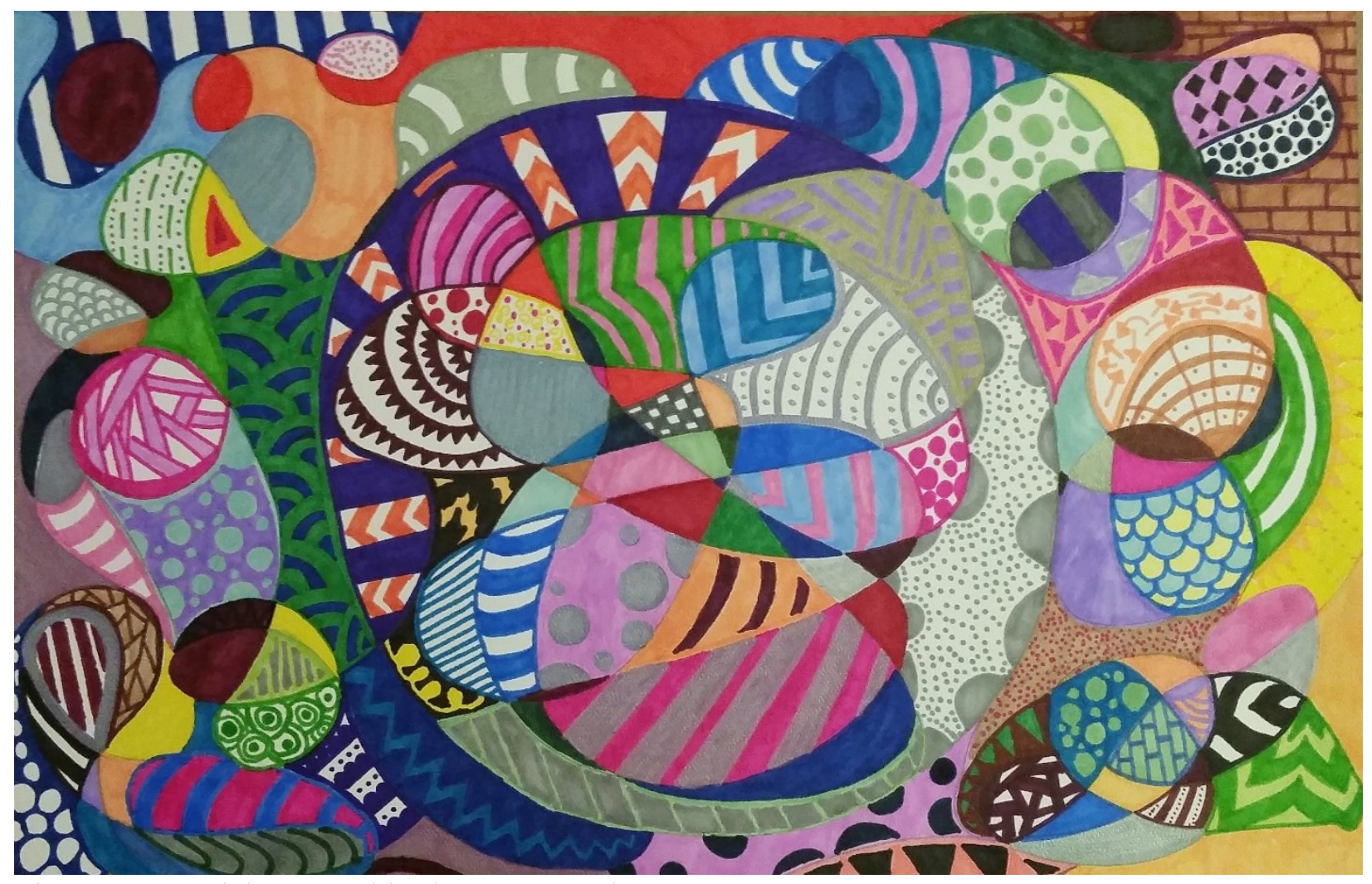

Figure 3. Mandala created in the Perceptual component. 


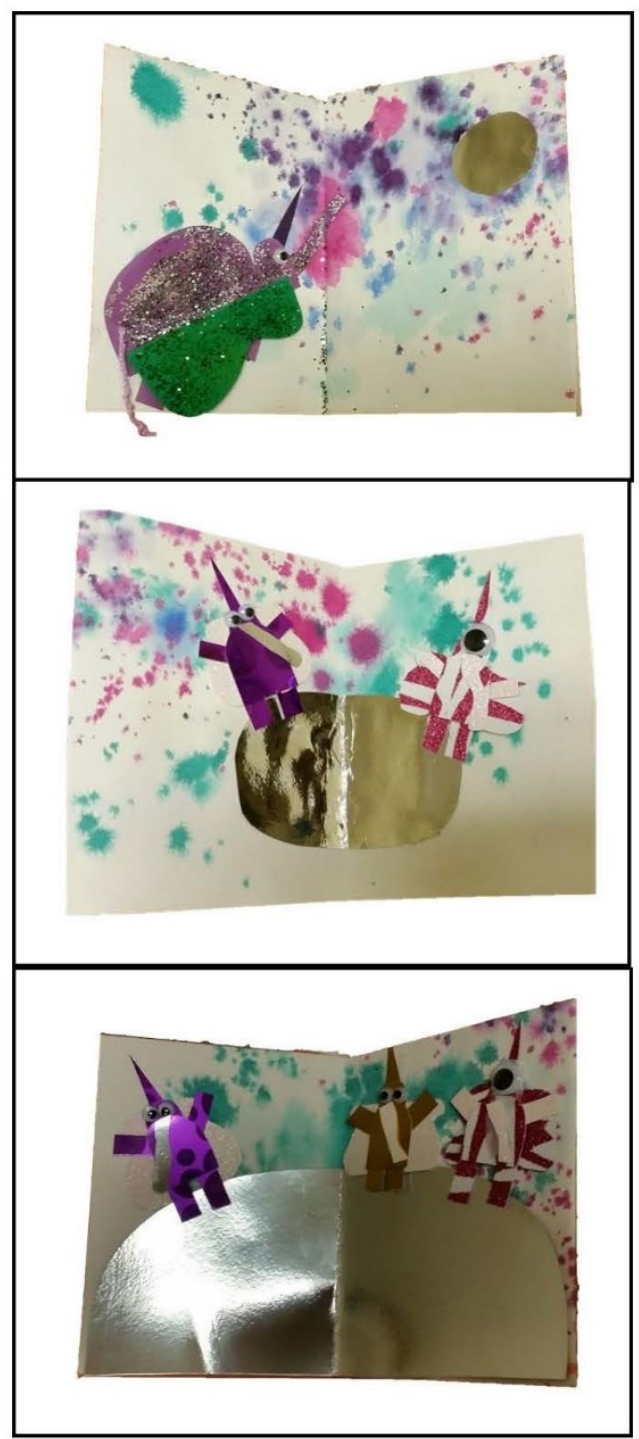

Figure 4. Excerpts of the book created in the Cognitive component. 


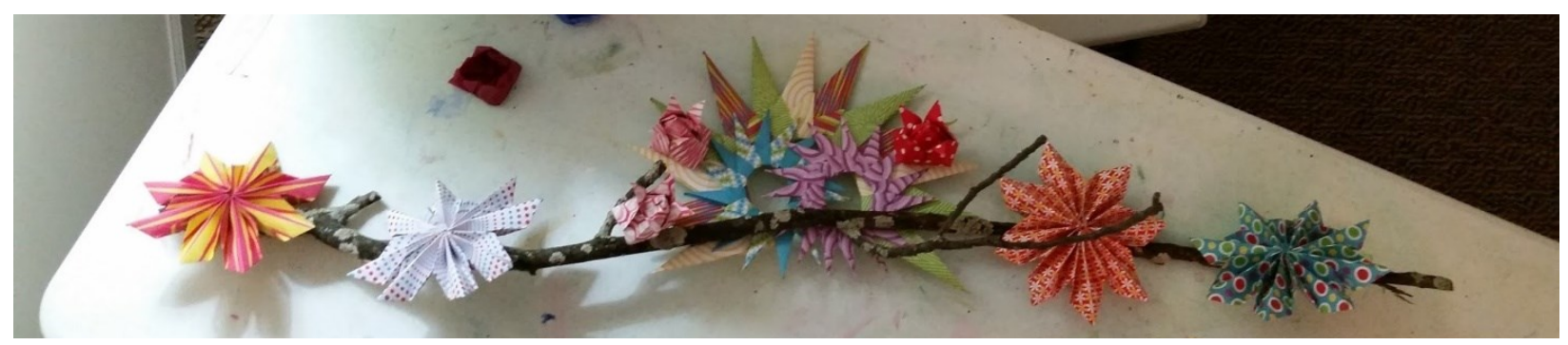

Figure 5. Origami flowers and stars created in the Sensory component. 


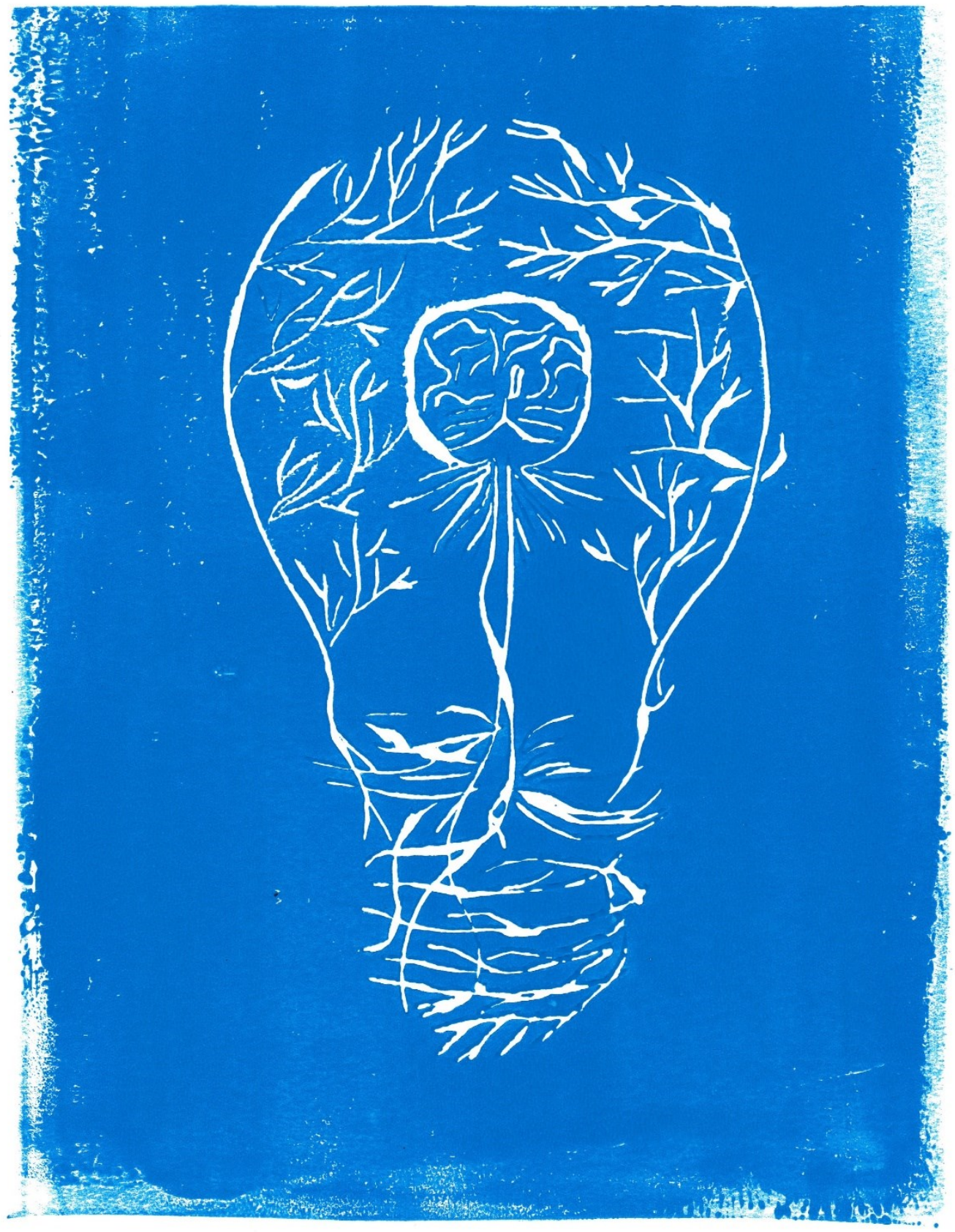

Figure 6. Self-symbol image carved and printed in the Symbolic component. 
Table 1. Alignment of the Areas of the Malnourished Brain and the ETC

\begin{tabular}{|c|c|c|c|c|c|c|c|c|c|}
\hline & PFC & A & PL & I & $\mathrm{H}$ & $\mathrm{SC}$ & CG & BG & NAC \\
\hline Kinesthetic & $\mathrm{X}$ & $\mathrm{X}$ & & & $\mathrm{X}$ & $X$ & $\mathrm{X}$ & $\mathrm{X}$ & $\mathrm{X}$ \\
\hline Sensory & $\mathrm{X}$ & & & $\mathrm{X}$ & & $\mathrm{X}$ & & & $\mathrm{X}$ \\
\hline Perceptual & $\mathrm{X}$ & & & $\mathrm{X}$ & $\mathrm{X}$ & & & & \\
\hline Affective & $\mathrm{X}$ & $\mathrm{X}$ & & & $\mathrm{X}$ & & $\mathrm{X}$ & & $\mathrm{X}$ \\
\hline Cognitive & $X$ & & $X$ & & $X$ & $X$ & $\mathrm{X}$ & & \\
\hline Symbolic & $\mathrm{X}$ & & & $\mathrm{X}$ & & & $\mathrm{X}$ & & \\
\hline
\end{tabular}

Note: prefrontal cortex (PFC), amygdala (A), parietal lobe (PL), insula (I), hippocampus (H), somatosensory cortex (SC), cingulate gyrus (CG), basal ganglia (BG), nucleus accumbens (NAC) 